\title{
Interacting Quintessence Dark Energy Models in Lyra Manifold
}

\author{
M. Khurshudyan, ${ }^{1}$ J. Sadeghi, ${ }^{2}$ R. Myrzakulov, ${ }^{3}$ Antonio Pasqua, ${ }^{4}$ and H. Farahani ${ }^{5}$ \\ ${ }^{1}$ Department of Theoretical Physics, Yerevan State University, 1 Alex Manookian, 0025 Yerevan, Armenia \\ ${ }^{2}$ Department of Physics, Islamic Azad University, Ayatollah Amoli Branch, P.O. Box 678, Amol, Iran \\ ${ }^{3}$ Department of Physics, Eurasian International Center for Theoretical Physics, Eurasian National University, \\ Astana 010008, Kazakhstan \\ ${ }^{4}$ Department of Physics, University of Trieste, Via Valerio, No. 2, 34127 Trieste, Italy \\ ${ }^{5}$ Department of Physics, Mazandaran University, P.O. Box 47416-95447, Babolsar, Iran
}

Correspondence should be addressed to H. Farahani; h.farahani@umz.ac.ir

Received 8 April 2014; Revised 8 August 2014; Accepted 13 August 2014; Published 1 September 2014

Academic Editor: Sally Seidel

Copyright (C) 2014 M. Khurshudyan et al. This is an open access article distributed under the Creative Commons Attribution License, which permits unrestricted use, distribution, and reproduction in any medium, provided the original work is properly cited. The publication of this article was funded by SCOAP ${ }^{3}$.

\begin{abstract}
We consider two-component dark energy models in Lyra manifold. The first component is assumed to be a quintessence field while the second component may be a viscous polytropic gas, a viscous Van der Waals gas, or a viscous modified Chaplygin gas. We also consider the possibility of interaction between components. By using the numerical analysis, we study some cosmological parameters of the models and compare them with observational data.
\end{abstract}

\section{Introduction}

Observations of high redshift type Supernovae Ia (SNeIa) [13] reveal the accelerated expansion of our universe, whose nature is not exactly clear until now. It is found that the density of matter is very much less than critical density [4]. Moreover, cosmic microwave background (CMB) radiation anisotropies observations indicate that the universe can be considered flat and the total energy density is very close to the critical value $\left(\Omega_{\text {tot }} \simeq 1\right)$ [5]. Based on the experimental data, a component of the energy dubbed as dark energy is thought to be responsible for the physics of the accelerated expansion but it seems that it is not alone in the universe, so the mysterious matter component which is called dark matter should also exist. Dark energy can be described by a pressure sufficiently negative in order to drive the acceleration of the universe and by positive energy density. There are several different models proposed to explain the nature of dark energy. The cosmological constant $\Lambda$ is the simplest model which can be considered, but in presence of many research papers in these fields, the origin of dark energy and dark matter is still unknown, and the possible connection between them is also unknown as well as real role of the components in the history of the universe. This situation gives a lot of freedom to researchers and possibility of some simulations. The cosmological constant faced two main problems, that is, the absence of a fundamental mechanism which sets the cosmological constant zero or very small value (which is known as fine-tuning problem) and the problem known as cosmological coincidence problem, which asks why we are living in an epoch in which the densities of dark energy and matter are comparable. One of the interesting ways to solve the above mentioned problems is to consider interactions between components [6]. From observational point of view, no piece of evidence has been so far presented against such interactions. Indeed, possible interactions between the components of universe have been discussed in recent years. It is found that a suitable interaction can help to alleviate the coincidence problem. Different interacting models of dark energy have been investigated [7-14].

Alternative models of dark energy suggest a dynamical form of dark energy, which, at least in an effective level, can originate from a variable cosmological constant $[15,16]$ or from various fields, such as a canonical scalar field [1721] (quintessence), a phantom field [20-22], or quintom [23-36]. Finally, interesting attempts to probe the nature of dark energy according to some basic quantum gravitational principles are the holographic dark energy paradigm [37-45] 
and agegraphic dark energy models [46, 47]. Among them a quintessence model is interesting in this paper as a component of dark energy. In that case, the dark energy may be dynamical approaching zero with time, or it may be slowly increasing. It is now dominating the universe because the reduction of mass and radiation energy density with the scale factor (which gives some information about the size of the universe) is greater than the decrease in dark energy density in the present epoch. In general, we would like the quintessence field to be decreasing with the scale factor and time at a smaller rate than the mass energy so that it will become dominant at redshifts less than one. The quintessence field has the property of being very weakly coupled to baryons but contributing a negative pressure to the equation of state. In the past it had a small contribution but with time it has decreased less quickly with the scale factor than the matter and radiation densities and is dominant now.

For the dark energy component we consider several models in this paper, including viscosity. Indeed, bulk viscosity is added to obtain more realistic models. However, viscous pressure can itself play the role of an agent that drives the present acceleration of the universe [48].

One of interesting dark energy models is the polytropic gas which was proposed to explain the accelerated expansion of the universe [49]. It was shown that the polytropic gas model in the presence of interaction can behave as phantom field [50]. It was pointed out that a polytropic scalar field can be reconstructed according to the evolutionary behaviors of the holographic and new agegraphic dark energy densities. The validity of the generalized second law of thermodynamics was also examined for the polytropic gas model in [51]. Another interesting model of dark energy may be Van der Waals gas which could be accounted as a fluid with unusual EoS or could be thought of as a fluid satisfying more general form of EoS; that is, $F(\rho, P)=0$ [52]. There are also some important models to describe dark energy based on Chaplygin gas equation of state which were recently considered by several papers such as [53-56] and yield good agreement with observational data.

On the other hand, the Lyra geometry provides one of the possible alternatives in modification of the cosmological models. As we know the modification of the gravitational theory has long been famous, but the late-time cosmological acceleration caused more research in this field [57]. Now, we like to consider a universe filled with a two-component dark energy in Lyra manifold with possibility of interaction between components. The first component is assumed to be quintessence, while we have several choices for the second component such as viscous polytropic gas, viscous Van der Waals gas, or viscous Chaplygin gas. We suggest these as toy models to describe universe and compare our results with observational data to choose one of them as the best model.

This paper is organized as follows. In Section 2, we introduce our models. In Section 3, we recall the main properties of field equations. In Section 4, we give numerical results corresponding to constant $\Lambda$. In Section 5, we give numerical results corresponding to varying $\Lambda$. In Section 6, we obtain some observational constraints. Finally, in Section 7, we write the conclusions of this paper.

\section{The Models}

One of the well studied dark energy models is the quintessence model $[17,18]$, which is a scalar field model described by a field $\phi$ and a $V(\phi)$ potential. It represents the simplest scalar field scenario without having theoretical problems such as the appearance of ghosts and Laplacian instabilities. The energy density $\rho_{\mathrm{Q}}$ and the pressure $P_{\mathrm{Q}}$ of the quintessence scalar field model are given, respectively, by

$$
\begin{aligned}
& \rho_{\mathrm{Q}}=\frac{1}{2} \dot{\phi}^{2}+V(\phi), \\
& P_{\mathrm{Q}}=\frac{1}{2} \dot{\phi}^{2}-V(\phi) .
\end{aligned}
$$

Canonical scalar field is not the unique solution. We can generalize it as follows [58]:

$$
\begin{aligned}
& \rho_{\mathrm{Q}}=\frac{\omega}{2} \phi^{k} \dot{\phi}^{2}+V(\phi), \\
& P_{\mathrm{Q}}=\frac{\omega}{2} \phi^{k} \dot{\phi}^{2}-V(\phi) .
\end{aligned}
$$

In the case of $k=0$, (2) transform to the canonical scalar field model with rescaling of the field. Below, we would like to consider an interaction term $Q$ between dark energy and dark matter described by

$$
Q=3 H b \rho_{Q}+\gamma\left(\rho_{b}-\rho_{Q}\right) \frac{\dot{\phi}}{\phi},
$$

where $b$ and $\gamma$ are positive constants with a typical value of $0.01-0.03$. Nature of the interaction between dark energy and dark matter is not clear. If we believe that it has a quantum origin, then an absence of the final theory of quantum gravity leaves this question as an open problem. However if we believe that the link existing between components is due to the same origin of the dark energy and dark matter, then this approach does not give any exact solution, because the nature of the two components is not formulated and it is another open problem. Therefore, only phenomenological assumption is an appropriate approach. For the dark matter model we will consider once a viscous modified Chaplygin gas with the following equation of state (EoS):

$$
P=A \rho-\frac{B}{\rho^{\alpha}}-3 \xi H,
$$

where $A, B$, and $\alpha$ are constants (with $0 \leq \alpha \leq 1$ in general relativity).

For the second model we will use viscous polytropic fluid with EoS given by

$$
P=K \rho^{1+1 / n}-3 \xi H,
$$

where $K$ is the polytropic index and $\xi$ represents the viscous coefficient.

In the third model we would like to consider interaction between quintessence dark energy and a viscous Van der Waals gas of the general form:

$$
P=\frac{A \rho}{B-\rho}-B \rho^{2}-3 \xi H,
$$


where $A$ and $B$ are constants. Furthermore, we will consider two regimes: (1) $\Lambda$ is a numerical constant; (2) $\Lambda$ is a function of the cosmic time $t$; therefore it is a varying quantity. In particular, we choose the following form for the time-varying $\Lambda$ :

$$
\Lambda(t)=H^{2} \phi^{-2}+\delta V(\phi),
$$

where $\delta$ is a positive constant and $V(\phi)$ is the potential of the field which we consider as follows:

$$
V(\phi)=V_{0} e^{\left(-\phi_{0} \phi\right)},
$$

where $\phi_{0}$ is a constant parameter.

We investigate the behavior of the cosmological parameters like the Hubble parameter $H$, deceleration parameter $q$, and EoS parameters of the quintessence dark energy and an effective two-component fluid. Moreover, we perform stability analysis via the squared speed of the sound $C_{S}^{2}$, which is defined as follows:

$$
C_{s}^{2}=\frac{\partial P}{\partial \rho}
$$

where $P$ and $\rho$ are, respectively, the pressure and the energy density of the effective fluid given by

$$
\begin{aligned}
& P=P_{Q}+P_{i}, \\
& \rho=\rho_{Q}+\rho_{i},
\end{aligned}
$$

where $i$ refers to one of the viscous fluids described above. We will finish our paper with the results obtained from observational constraints. Consideration of the statefinder analysis, different forms of interaction terms, and different $\Lambda(t)$ and varying viscosity is possible and an interesting research. We hope to approach this question in future with forthcoming articles.

\section{The Field Equations}

The field equations governing our model are given by

$$
R_{\mu \nu}-\frac{1}{2} g_{\mu \nu} R-\Lambda g_{\mu \nu}+\frac{3}{2} \phi_{\mu} \phi_{\nu}-\frac{3}{4} g_{\mu \nu} \phi^{\alpha} \phi_{\alpha}=T_{\mu \nu}
$$

Considering the content of the universe to be a perfect fluid, we have

$$
T_{\mu \nu}=(\rho+P) u_{\mu} u_{\nu}-P g_{\mu \nu},
$$

where $u_{\mu}=(1,0,0,0)$ is the 4 -velocity of the comoving observer, satisfying the relation $u_{\mu} u^{\mu}=1$. Let $\phi_{\mu}$ be a timelike vector field of displacement; then

$$
\phi_{\mu}=\left(\frac{2}{\sqrt{3}} \beta, 0,0,0\right),
$$

where $\beta=\beta(t)$ is a function of time alone and the factor $2 / \sqrt{3}$ is inserted in order to simplify the writing of all the following equations. By using FRW metric for a flat universe given by

$$
d s^{2}=-d t^{2}+a(t)^{2}\left(d r^{2}+r^{2} d \Omega^{2}\right),
$$

field equations can be reduced to the following Friedmann equations:

$$
\begin{gathered}
3 H^{2}-\beta^{2}=\rho+\Lambda, \\
2 \dot{H}+3 H^{2}+\beta^{2}=-P+\Lambda,
\end{gathered}
$$

where $H=\dot{a} / a$ is the Hubble parameter, the dot stands for differentiation with respect to the cosmic time $t, d \Omega^{2}=$ $d \theta^{2}+\sin ^{2} \theta d \phi^{2}$, and $a(t)$ represents the scale factor. The $\theta$ and $\phi$ parameters are the usual azimuthal and polar angles of spherical coordinates, with $0 \leq \theta \leq \pi$ and $0 \leq \phi<2 \pi$. The coordinates $(t, r, \theta, \phi)$ are called comoving coordinates.

The continuity equation is given by

$$
\dot{\rho}+\dot{\Lambda}+2 \beta \dot{\beta}+3 H\left(\rho+P+2 \beta^{2}\right)=0 .
$$

The continuity equation given in (16) can be also rewritten in the compact form:

$$
\dot{\rho}+3 H(\rho+P)=0 .
$$

Comparing (16) and (17) we obtain the following link between $\Lambda$ and $\beta$ :

$$
\dot{\Lambda}+2 \beta \dot{\beta}+6 H \beta^{2}=0
$$

In order to introduce an interaction between dark energy and dark matter, we should mathematically split (17) into the two following equations:

$$
\begin{gathered}
\dot{\rho}_{i}+3 H\left(\rho_{i}+P_{i}\right)=Q, \\
\dot{\rho}_{\mathrm{Q}}+3 H\left(\rho_{\mathrm{Q}}+P_{\mathrm{Q}}\right)=-\mathrm{Q} .
\end{gathered}
$$

The cosmological parameters of our interest are the EoS parameter of each fluid component $\omega_{i}=P_{i} / \rho_{i}$, the EoS parameter of composed fluid,

$$
\omega_{\text {tot }}=\frac{P_{Q}+P_{i}}{\rho_{Q}+\rho_{i}},
$$

and the deceleration parameter $q$, which can be written as follows:

$$
q=\frac{1}{2}\left(1+3 \frac{P}{\rho}\right)
$$

where index $i$ refers to the first components which is viscous modified Chaplygin gas or viscous polytropic fluid and index $Q$ refers to the quintessence scalar field. A differential equation describing dynamics of the DE after some mathematics can be rewritten as

$$
\dot{\rho}_{\mathrm{Q}}+3 H \rho_{\mathrm{Q}}\left(1+b+\omega_{\mathrm{Q}}-\frac{\gamma}{3 H} \frac{\dot{\phi}}{\phi}\right)=-\gamma \rho_{i} \frac{\dot{\phi}}{\phi} .
$$

Taking into account the form of the varying $\Lambda(t)$ from (7) for the Hubble parameter $H$ we will have

$$
H=\frac{1}{\sqrt{3}} \sqrt{\frac{\rho+\delta e^{\left[-\phi_{0} \phi\right]}+\beta^{2}}{1-\phi^{-2} / 3}} .
$$

Hereafter, we will consider $\phi_{0}=1$ for mathematical simplicity. 


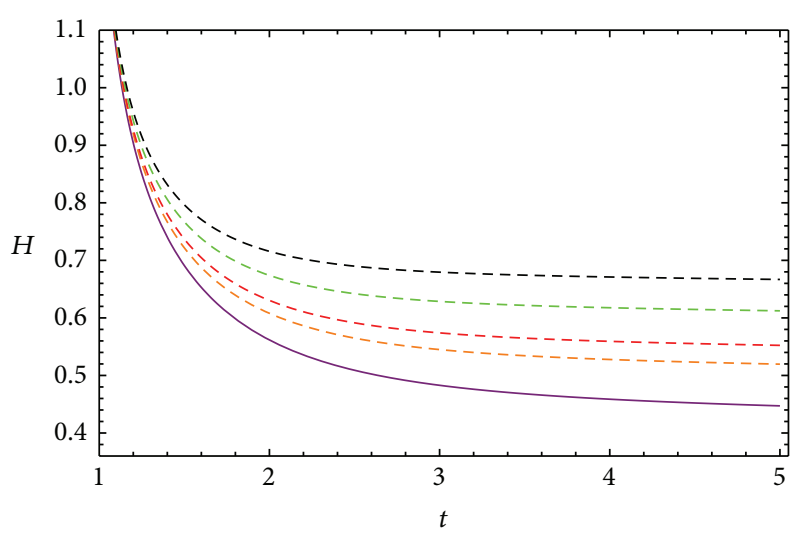

$\gamma=0.02, b=0.01$ and $\xi=0.1$
$-\{\Lambda=0 ., \alpha=0.5, A=2.5\}$
$--\{\Lambda=0.2, \alpha=0.5, A=2.5\}$
$---\{\{\Lambda=0.5, \alpha=0.5, A=2.5\}$
$--\{\Lambda=0.3, \alpha=0.5, A=2.5\}$

(a)

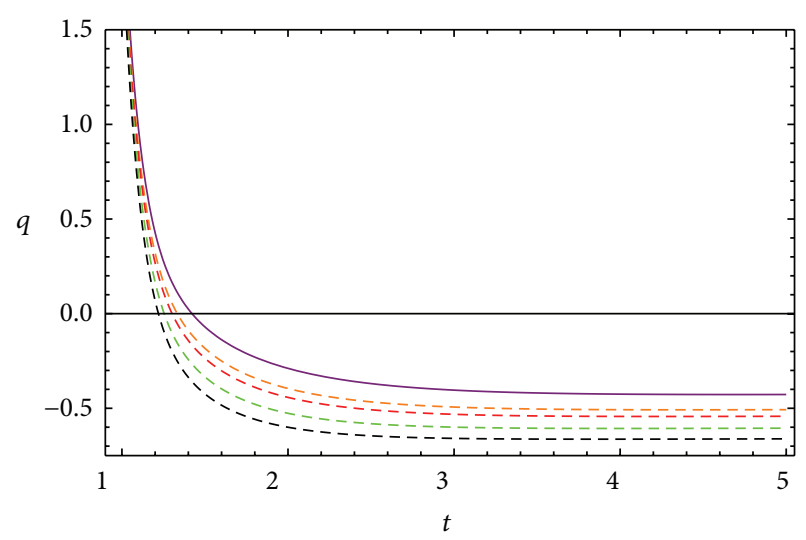

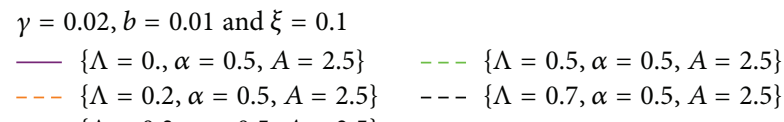
$---\{\Lambda=0.3, \alpha=0.5, A=2.5\}$

(b)

Figure 1: Behavior of Hubble parameter $H$ and $q$ against $t$ for the constant $\Lambda$. Model 1.

\section{Case of Constant $\Lambda$}

We found it reasonable to start our analysis from the models with constant $\Lambda$. Without loss of generality, we would like to describe equations allowing us to find dynamics of the models. According to the assumption with constant $\Lambda$ (16) will be modified as follows:

$$
\dot{\rho}+2 \beta \dot{\beta}+3 H\left(\rho+P+2 \beta^{2}\right)=0
$$

and, using the expression $\dot{\rho}+3 H(\rho+P)=0$, we will obtain that

$$
\dot{\beta}+3 H \beta=0
$$

The last equation can be integrated very easily and the result is the following:

$$
\beta=\beta_{0} a^{-3}
$$

where $a(t)$ is the scale factor and $\beta_{0}$ is the integration constant. In our future calculations we will use $\beta_{0}=1$ as initial condition. For the Hubble parameter $H$ we will obtain

$$
H=\frac{1}{\sqrt{3}} \sqrt{\rho+\Lambda+\beta_{0} a^{-6}}
$$

Concerning the form of the field equations, we need only to assume the form of $Q$ and we will obtain the cosmological solutions. Concerning the mathematical hardness of the problem we will analyze models numerically and investigate graphical behavior of various important cosmological parameters with respect to the cosmic time. In the following subsections we consider three models with the particular form of $Q$ with given forms of the EoS for the viscous dark matter fluids considered in the Introduction.
4.1. Model 1. The first toy model describes the dynamics of the universe within an effective fluid in case of the cosmological constant. The dynamics of the energy density of the viscous modified Chaplygin gas which will model dark matter in our universe and the differential equation describing the dynamics of it can be found to be

$$
\begin{aligned}
\dot{\rho}_{\mathrm{Ch}} & +3 H\left(1+A-\frac{B}{\rho_{\mathrm{Ch}}^{\alpha+1}}-\frac{\gamma}{3 H} \frac{\dot{\phi}}{\phi}\right) \rho_{\mathrm{Ch}} \\
& =3 H\left(b-\frac{\gamma}{3 H} \frac{\dot{\phi}}{\phi}\right) \rho_{\mathrm{Q}}+9 \xi H .
\end{aligned}
$$

From (23) for the dynamics of the dark energy, we have

$$
\dot{\rho}_{\mathrm{Q}}+3 H\left(1+b+\omega_{\mathrm{Q}}-\frac{\gamma}{3 H} \frac{\dot{\phi}}{\phi}\right) \rho_{\mathrm{Q}}=-\gamma \frac{\dot{\phi}}{\phi} \rho_{\mathrm{Ch}} .
$$

The best fit for the theoretical model of our consideration with the observational data we obtained for $H_{0}=1.4, \Omega_{\mathrm{Ch}}=0.3$, $\Lambda=0.7, A=2.5, B=0.9, \gamma=0.02, b=0.01$, and $\xi=0.1$.

In Figures 1-3, we have chosen to plot some of the quantities derived for different values of the parameters involved. In particular, we have chosen $\gamma=0.02, b=0.01$, $\xi=0.1, \alpha=0.5$, and $A=2.5$ and we have chosen five different values of $\Lambda$, that is, $0,0.2,0.3,0.5$, and 0.7 .

Figure 1 shows that the Hubble parameter $H$ is decreasing with time to a constant at the late universe as expected and its value increased by $\Lambda$. On the other hand, the value of the deceleration parameter decreased with $\Lambda$. It is illustrated by the right plot of Figure 1 . For $\Lambda=0$ we can see $q \rightarrow-0.4$, while for $\Lambda=0.7$ we can see $q \rightarrow-0.7$. Also acceleration to deceleration phase transition is seen in this model. At the late time we have $q \sim-0.5$ being in agreement with observational data. As we know recent observations of type SNIa indicate that universe is accelerating with the deceleration parameter lying somewhere in the range $-1<q \leq 0$. 

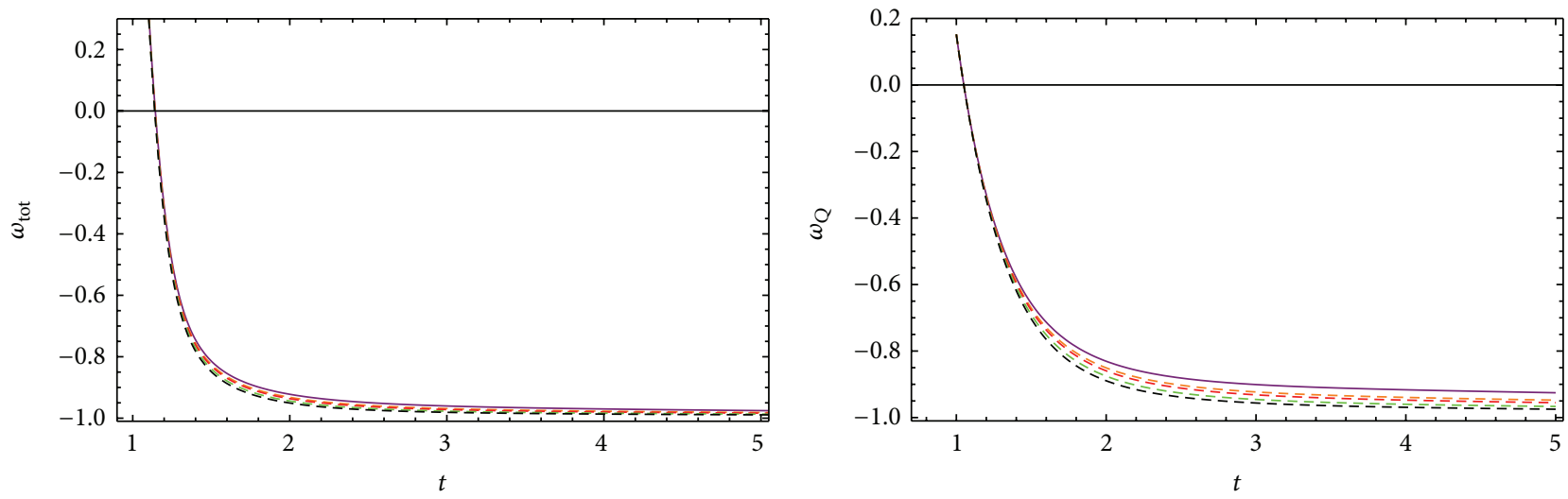

$$
\begin{aligned}
& \gamma=0.02, b=0.01 \text { and } \xi=0.1 \\
& -\{\Lambda=0 ., \alpha=0.5, A=2.5\} \\
& ---\{\Lambda=0.2, \alpha=0.5, A=2.5\} \\
& --\quad\{\Lambda=0.3, \alpha=0.5, A=2.5\}
\end{aligned}
$$

(a)

$$
\begin{aligned}
& \gamma=0.02, b=0.01 \text { and } \xi=0.1 \\
& -\{\Lambda=0 ., \alpha=0.5, A=2.5\} \\
& ---\{\Lambda=0.2, \alpha=0.5, A=2.5\} \\
& ---\{\Lambda=0.5, \alpha=0.5, A=2.5\} \\
& --\{\Lambda=0.3, \alpha=0.5, A=2.5\}
\end{aligned}
$$

(b)

FIgURE 2: Behavior of EoS parameters $\omega_{\text {tot }}$ and $\omega_{\mathrm{Q}}$ against $t$ for the constant $\Lambda$. Model 1 .

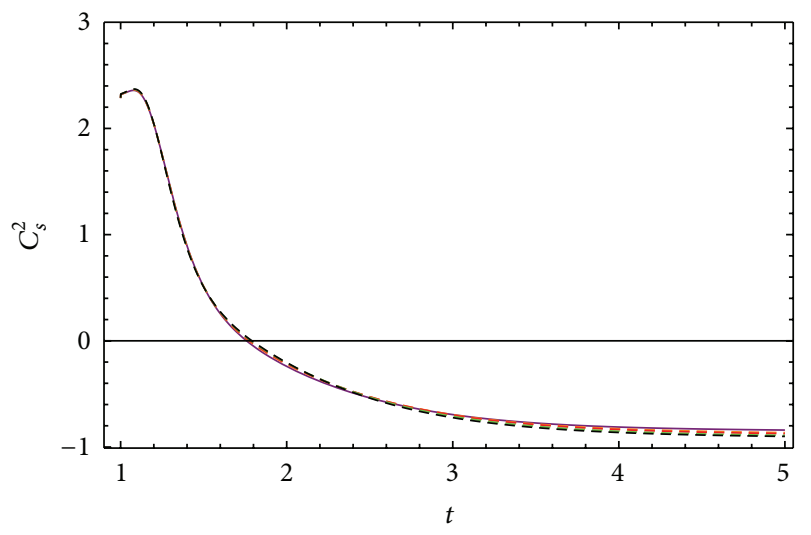

$\gamma=0.02, b=0.01$ and $\xi=0.1$

$\begin{aligned}\{\Lambda=0 ., \alpha=0.5, A=2.5\} & --\{\Lambda=0.5, \alpha=0.5, A=2.5\} \\ --\{\Lambda=0.2, \alpha=0.5, A=2.5\} & --\{\Lambda=0.7, \alpha=0.5, A=2.5\}\end{aligned}$

Figure 3: Squared sound speed against $t$ for the constant $\Lambda$. Model 1.

Moreover, Figure 2 shows that the EoS parameter tends to -1 at the late time with $\omega_{\text {tot }} \geq-1$, corresponding to a quintessence-like universe.

Unfortunately, analysis of squared sound speed (see Figure 3) shows that this model is not stable at the late time and will be considered only for the early universe.

4.2. Model 2. In the second model, after some mathematical calculations, we obtain the following differential equation to study dynamics of the model:

$$
\begin{aligned}
\dot{\rho}_{P} & +3 H\left(1+\omega_{P}-\frac{\gamma}{3 H} \frac{\dot{\phi}}{\phi}\right) \rho_{P} \\
& =3 H\left(b-\frac{\gamma}{3 H} \frac{\dot{\phi}}{\phi}\right) \rho_{\mathrm{Q}}+9 \xi H,
\end{aligned}
$$

where $\omega_{P}$ is given as

$$
\omega_{P}=K \rho_{P}^{1 / n}-\frac{3 \xi H}{\rho_{P}}
$$

The best fitted values of parameters are $H_{0}=1.2, \Omega_{P}=0.25$, $\Lambda=1.5, K=2.5, n=1.0, \gamma=0.02, b=0.01$, and $\xi=0.2$.

In Figures 4 and 5, we have chosen to plot some of the quantities derived for different values of the parameters involved. In particular, we have chosen $\gamma=0.02, b=0.01$, $\xi=0.1, n=1$, and $K=2.5$ and we have chosen five different values of $\Lambda$, that is, $0,0.5,1.0,1.2$, and 1.5 .

Numerical results of the Hubble expansion parameter and the deceleration parameter $q$ show good behavior (see Figure 4), but the stability analysis illustrated in Figure 5 shows that this model also has instability in the late time and is only useful for the early universe. However, the effect of constant $\Lambda$ in this parameter is similar to the first model and the EoS parameter tends to -1 as before. In the case of $\Lambda=0$, we can see that $q \sim-0.2$, which is not coincident with observational data. It tells that presence of $\Lambda$ may be necessary to obtain agreement with observations.

Figure 5 shows that instability of model may solve for the large value of the cosmological constant. Therefore model 2 is stable at the late time for the large value of the $\Lambda$.

4.3. Model 3. In the third model we have the following expression for the pressure $P$ :

$$
P=\frac{A \rho_{W}}{B-\rho_{W}}-B \rho_{W}^{2}-3 \xi H .
$$

The constant $\Lambda$ assumption will lead us to the following expression for the Hubble parameter $H$ :

$$
H=\frac{1}{\sqrt{3}} \sqrt{\rho+\Lambda+\beta_{0} a^{-6}} .
$$




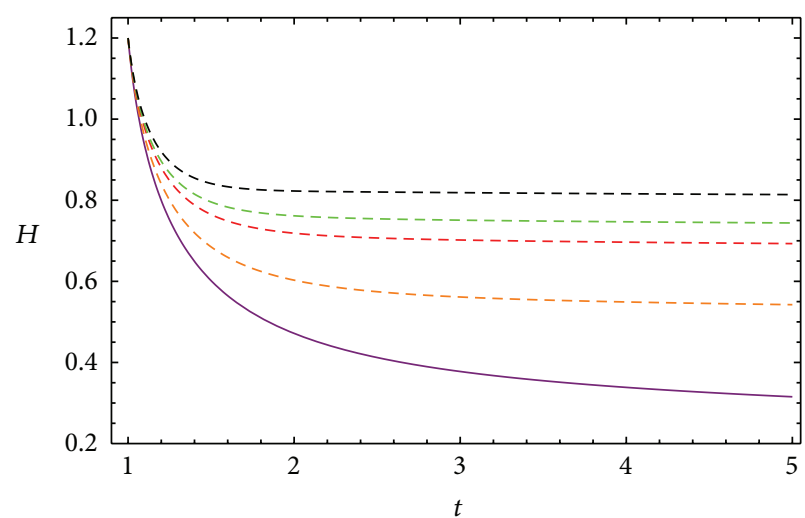

$$
\begin{aligned}
& \gamma=0.02, b=0.01 \text { and } \xi=0.2 \\
& -\{\Lambda=0 ., n=1 ., K=2.5\} \\
& ---\{\Lambda=0.5, n=1 ., K=2.5\} \\
& ---\{\Lambda=1 ., n=1 ., K=2.5\}
\end{aligned}
$$

$$
\begin{aligned}
& --\{\Lambda=1.2, n=1 ., K=2.5\} \\
& --\{\Lambda=1.5, n=1 ., K=2.5\}
\end{aligned}
$$

(a)

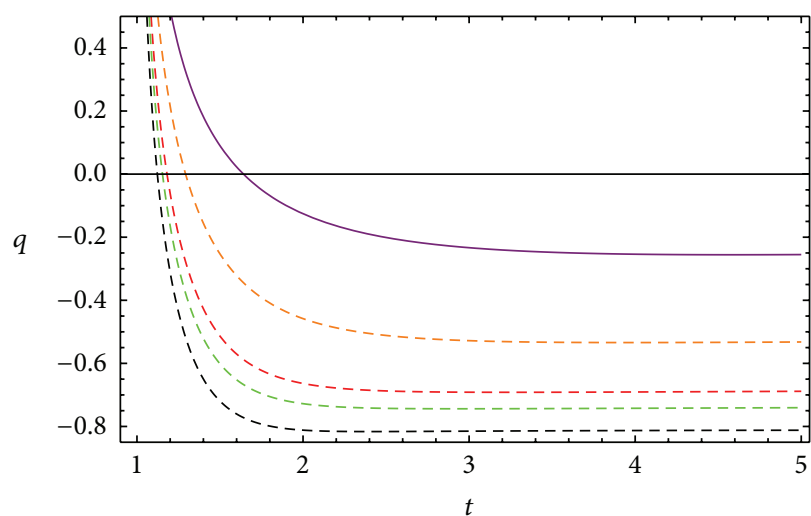

$\gamma=0.02, b=0.01$ and $\xi=0.2$

$-\{\Lambda=0 ., n=1 ., K=2.5\}$

$\{\Lambda=0.5, n=1 ., K=2.5\}$

$---\{\Lambda=1 ., n=1 ., K=2.5\}$
$-\ldots\{\Lambda=1.5, n=1 ., K=2.5\}$

(b)

FIGURE 4: Behavior of Hubble parameter $H$ and $q$ against $t$ for the constant $\Lambda$. Model 2.

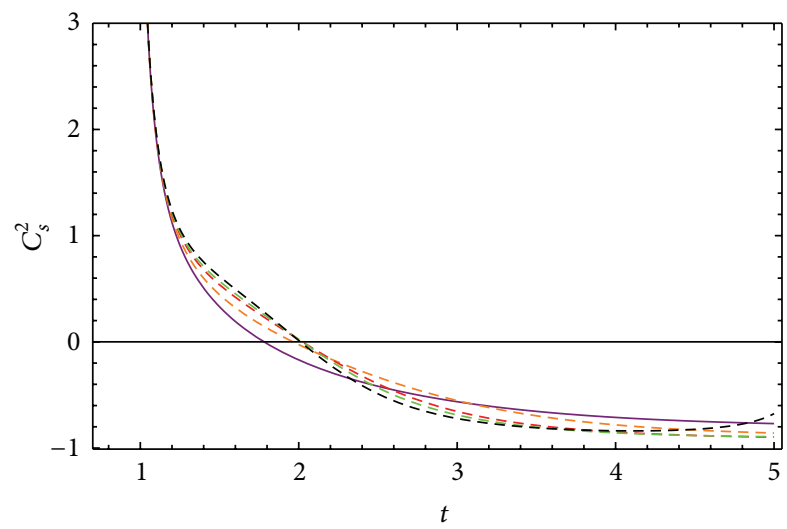

$\gamma=0.02, b=0.01$ and $\xi=0.2$

$-\{\Lambda=0 ., n=1 ., K=2.5\}$

$---\{\Lambda=0.5, n=1 ., K=2.5\}$

$---\{\Lambda=1 ., n=1 ., K=2.5\}$

FIgURE 5: Squared sound speed against $t$ for the constant $\Lambda$. Model 2 .

The differential equations describing the dynamics of the energy densities of both components are given by the following equations:

$$
\begin{aligned}
\dot{\rho}_{W} & +3 H \rho_{W}\left(1+\frac{A}{B-\rho_{W}}-B \rho_{W}-\frac{\gamma}{3 H} \frac{\dot{\phi}}{\phi}\right) \\
& =3 H \rho_{\mathrm{Q}}\left(b-\frac{\gamma}{3 H} \frac{\dot{\phi}}{\phi}\right)+9 \xi H, \\
\dot{\rho}_{\mathrm{Q}} & +3 H \rho_{\mathrm{Q}}\left(1+b+\omega_{\mathrm{Q}}-\frac{\gamma}{3 H} \frac{\dot{\phi}}{\phi}\right)=-\gamma \rho_{W} \frac{\dot{\phi}}{\phi},
\end{aligned}
$$

where $\omega_{Q}$ is the EoS parameter of dark energy. The best fit for the theoretical model of our consideration with the

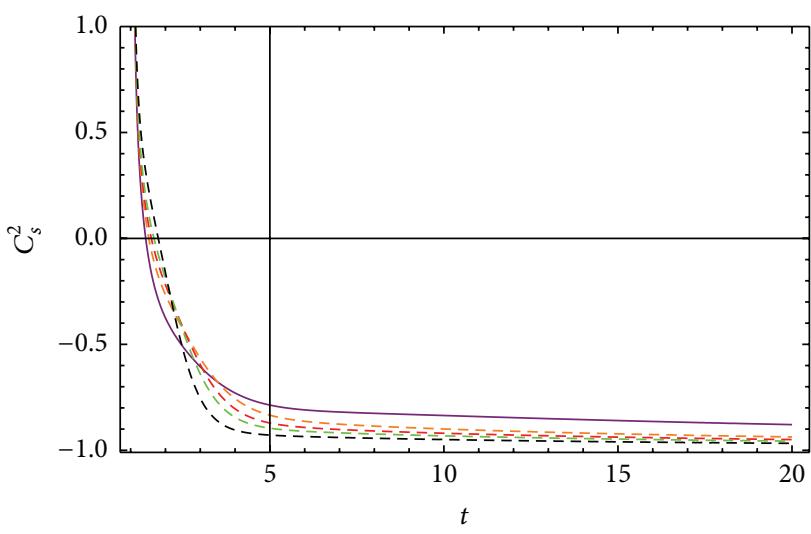

$$
\begin{aligned}
& \gamma=0.02, b=0 . \text { and } \xi=0.4 \\
& -\{\Lambda=0 ., A=1.5, B=1.2\} \\
& ---\{\Lambda=0.3, A=1.5, B=1.2\} \quad---\{\Lambda=0.7, A=1.5, B=1.2\} \\
& ---\{\Lambda=0.5, A=1.5, B=1.2\}
\end{aligned}
$$

Figure 6: Squared sound speed against $t$ for the constant $\Lambda$. Model 3.

observational data is obtained for $H_{0}=1.3, \Omega_{P}=0.217$, $\Lambda=1.2, A=1.5, B=1.2, \gamma=0.02, b=0.00$, and $\xi=0.4$.

The behaviors of the cosmological parameters are similar to the previous models and we can see late time instability of this model in Figure 6 . This suggests considering varying $\Lambda$ to obtain more appropriate models.

\section{The Case of Varying $\Lambda$}

In this section we will consider three interacting fluid models and will investigate cosmological parameters like the Hubble parameter $H$, deceleration parameter $q$, and EoS parameters of the total fluid and dark energy $\omega_{Q}$. Based on numerical solutions, we will discuss graphical behaviors of 


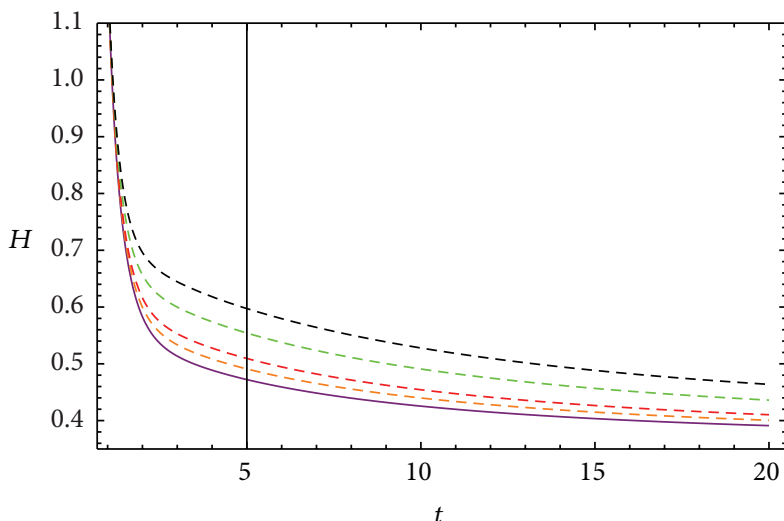

$\gamma=0.02, b=0.01$ and $\xi=0.5$

$-\{\delta=0.1, \alpha=0.5, A=1.5\}$

$--\{\delta=0.3, \alpha=0.5, A=1.5\}$

$--\{\delta=0.5, \alpha=0.5, A=1.5\}$

(a)

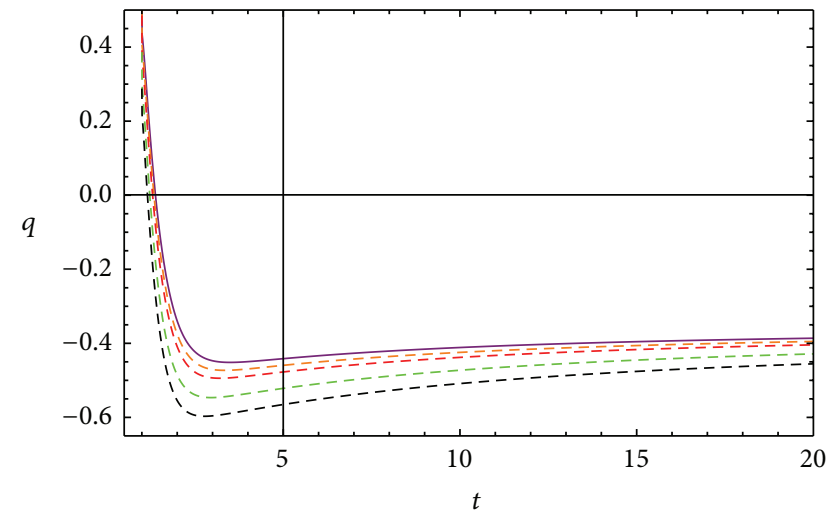

$\gamma=0.02, b=0.01$ and $\xi=0.5$
$-\{\delta=0.1, \alpha=0.5, A=1.5\}$
$---\{\delta=0.3, \alpha=0.5, A=1.5\}$
$----\{\delta=1 ., \alpha=0.5, A=1.5\}$
$-\{\delta=0.5, \alpha=0.5, A=1.5\}$

(c)

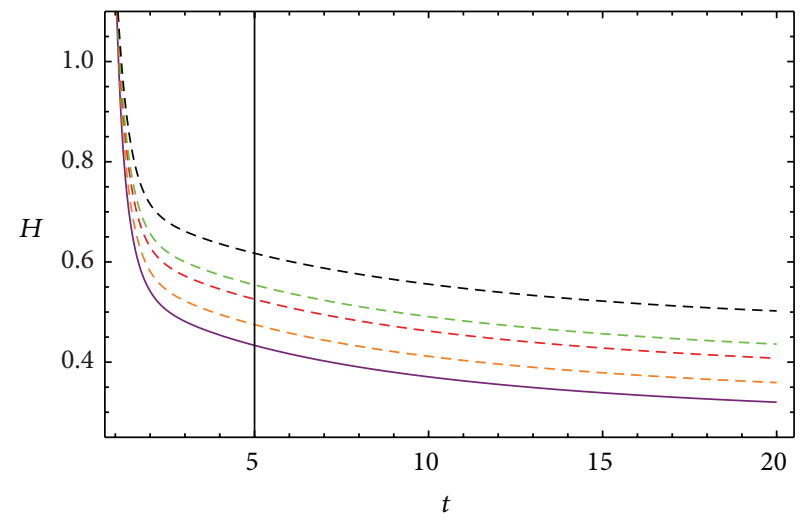

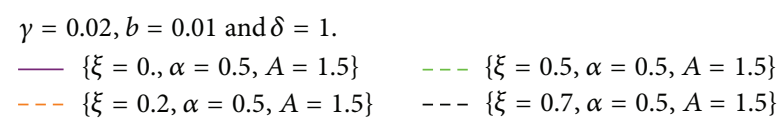

(b)

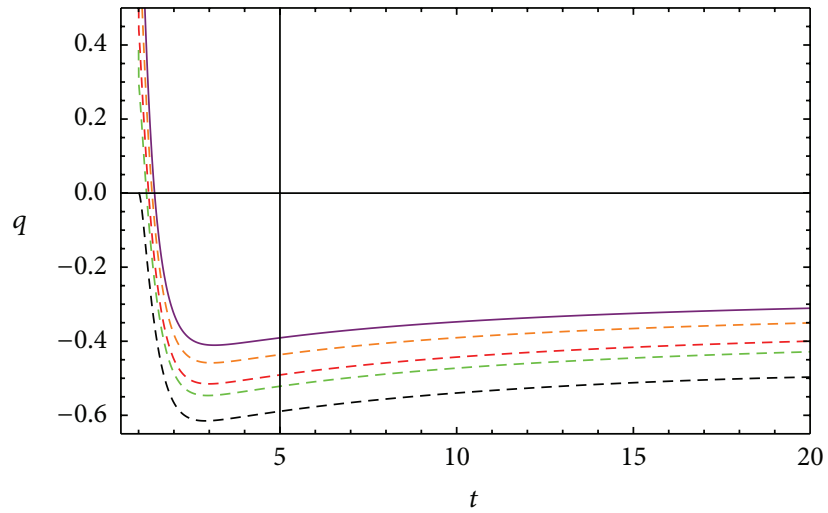

$\gamma=0.02, b=0.01$ and $\delta=1$

$\{\xi=0 ., \alpha=0.5, A=1.5\}$

- - $\{\xi=0.5, \alpha=0.5, A=1.5\}$

$---\{\xi=0.2, \alpha=0.5, A=1.5\}$

$--\{\xi=0.4, \alpha=0.5, A=1.5\}$

(d)

FIGURE 7: Behavior of Hubble parameter $H$ and deceleration parameter $q$ against $t$ for varying $\Lambda$. Model 4 .

the cosmological parameters. For the varying $\Lambda$ we take a phenomenological form which was considered by us recently [59]. The formula of $\Lambda$ is given as the following expression:

$$
\Lambda(t)=H^{2} \phi^{-2}+\delta V(\phi),
$$

which is a function of the Hubble parameter, potential of the scalar field, and time derivative of the scalar field. For the potential we take a simple form $V(\phi)=e^{[-\phi]}$; therefore the form of $\Lambda$ can be written also in the following way as only a function of the filed $\phi$ :

$$
\Lambda(t)=H^{2} \phi^{-2}+\delta e^{-\phi}
$$

Therefore, the dynamics of $\beta$ can be obtained from the following differential equation:

$$
2 \beta \dot{\beta}+6 H \beta^{2}+2 H \dot{H} \phi^{-2}-2 H^{2} \phi^{-3} \dot{\phi}-\delta e^{[-\phi]} \dot{\phi}=0 .
$$

In forthcoming subsections within three different forms of $Q$ we will investigate the dynamics of the universe. The question of the dynamics for the energy densities of the dark energy and dark matter is already discussed in a previous section; therefore we will not consider them here and we will start with the comments on the graphical behaviors of the cosmological parameters of the models. We will start with the model where

$$
Q=3 H b \rho_{Q}+\gamma\left(\rho_{i}-\rho_{Q}\right) \frac{\dot{\phi}}{\phi} .
$$

5.1. Model 4. Interacting viscous modified Chaplygin gas with the quintessence dark energy in the case of varying $\Lambda$ gives the following differential equation:

$$
\begin{aligned}
\dot{\rho}_{\mathrm{Ch}} & +3 H\left(1+A-\frac{B}{\rho_{\mathrm{Ch}}^{\alpha+1}}-\frac{\gamma}{3 H} \frac{\dot{\phi}}{\phi}\right) \rho_{\mathrm{Ch}} \\
& =3 H\left(b-\frac{\gamma}{3 H} \frac{\dot{\phi}}{\phi}\right) \rho_{\mathrm{Q}}+9 \xi H,
\end{aligned}
$$



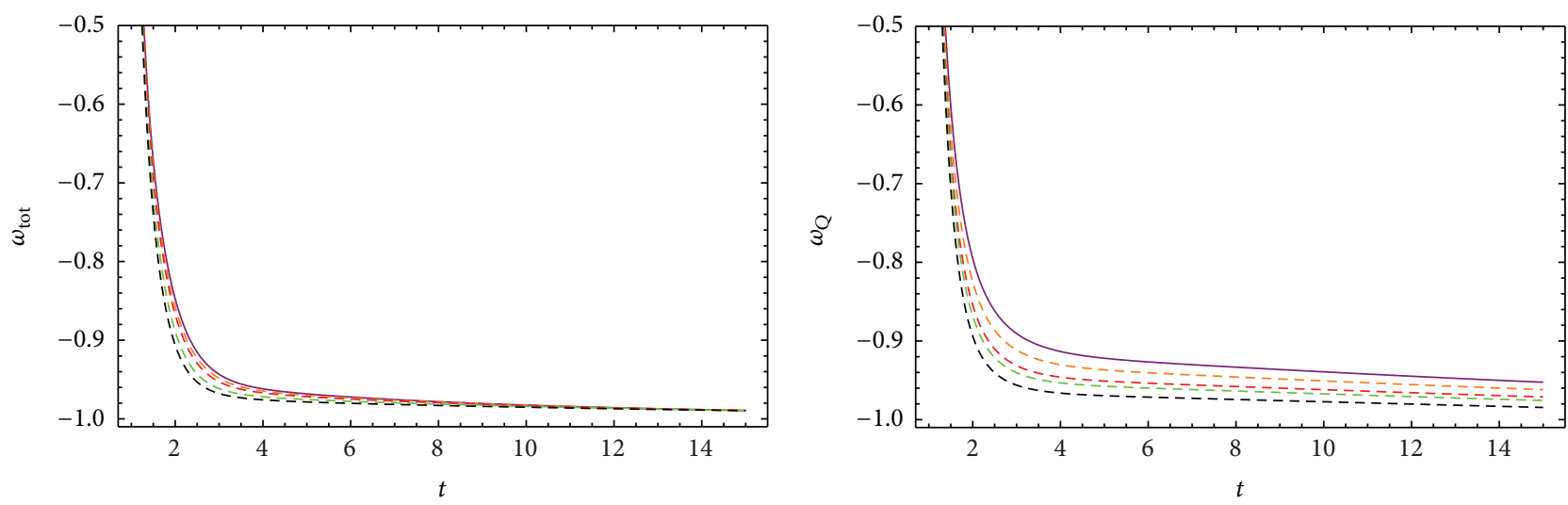

$$
\begin{aligned}
& \gamma=0.02, b=0.01 \text { and } \xi=0.5 \\
& -\{\delta=0.1, \alpha=0.5, A=1.5\} \\
& \text { - - }\{\delta=0.3, \alpha=0.5, A=1.5\} \\
& ---\{\delta=0.5, \alpha=0.5, A=1.5\} \\
& --\{\delta=1 ., \alpha=0.5, A=1.5\} \\
& \text { - - }\{\delta=1.5, \alpha=0.5, A=1.5\}
\end{aligned}
$$

(a)

$$
\begin{aligned}
& \gamma=0.02, b=0.01 \text { and } \delta=1 \text {. } \\
& \text { - }\{\xi=0 ., \alpha=0.5, A=1.5\} \quad-\ldots\{\xi=0.5, \alpha=0.5, A=1.5\} \\
& --\{\xi=0.2, \alpha=0.5, A=1.5\} \quad--\{\xi \xi=0.7, \alpha=0.5, A=1.5\}
\end{aligned}
$$$$
--\{\xi=0.4, \alpha=0.5, A=1.5\}
$$

(b)

FIgURE 8: Behavior of the EoS parameters $\omega_{\text {tot }}$ and $\omega_{\mathrm{Q}}$ against $t$ for the case corresponding to varying $\Lambda$. Model 4 .

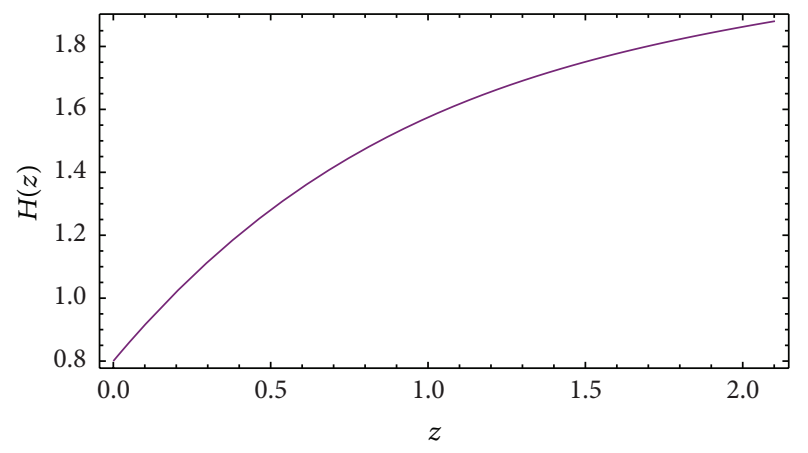

Figure 9: Hubble parameter $H$ against redshift $z$ for varying $\Lambda$. Model 4.

with the Hubble parameter obtained as

$$
H=\frac{1}{\sqrt{3}} \sqrt{\frac{\rho+\delta e^{\left[-\phi_{0} \phi\right]}+\beta^{2}}{1-\phi^{-2} / 3}}
$$

In Figure 7, we can see that the Hubble expansion parameter is a decreasing function of time $t$, which yields a constant at the late time as expected. It is clear that $\delta$ and $\xi$ increase the value of the Hubble expansion parameter but decrease the value of the deceleration parameter. In order to obtain the deceleration parameter being in agreement with observational data, we should choose larger values of $\delta$ and $\xi$. Also acceleration to deceleration phase transition happens in this model. We find an instability at the initial time but the model is completely stable at the late time.

Figure 8 shows that the EoS parameters yield -1 at the late time, in agreement with observational data. Also effects of $\delta$ and $\xi$ are illustrated in the plots of Figure 8.
In Figure 9, we can see behavior of the Hubble expansion parameter with the redshift which is also in agreement with observational data, since it is increasing function.

5.2. Model 5. A polytropic fluid interacting with the quintessence dark energy yields the following differential equation:

$$
\begin{aligned}
\dot{\rho}_{P} & +3 H\left(1+\omega_{P}-\frac{\gamma}{3 H} \frac{\dot{\phi}}{\phi}\right) \rho_{P} \\
& =3 H\left(b-\frac{\gamma}{3 H} \frac{\dot{\phi}}{\phi}\right) \rho_{Q}+9 \xi H,
\end{aligned}
$$

with the Hubble parameter obtained as

$$
H=\frac{1}{\sqrt{3}} \sqrt{\frac{\rho+\delta e^{\left[-\phi_{0} \phi\right]}+\beta^{2}}{1-\phi^{-2} / 3}}
$$

In Figure 10, we can see behavior of the Hubble expansion parameter and the deceleration parameter with time and find that the value of $K$ decreases the value of the Hubble expansion parameter while it increases the value of the deceleration parameter. Also, acceleration to deceleration phase transition is seen in this model.

However, squared sound speed plotted in Figure 11 shows that this model, similar to the constant $\Lambda$, is completely instable at the late time and will be useful only for the early universe.

5.3. Model 6. Finally, in the third model for the case of varying $\Lambda$ where a viscous Van der Waals gas with EoS of the general form

$$
P=\frac{A \rho_{W}}{B-\rho_{W}}-B \rho_{W}^{2}-3 \xi H .
$$



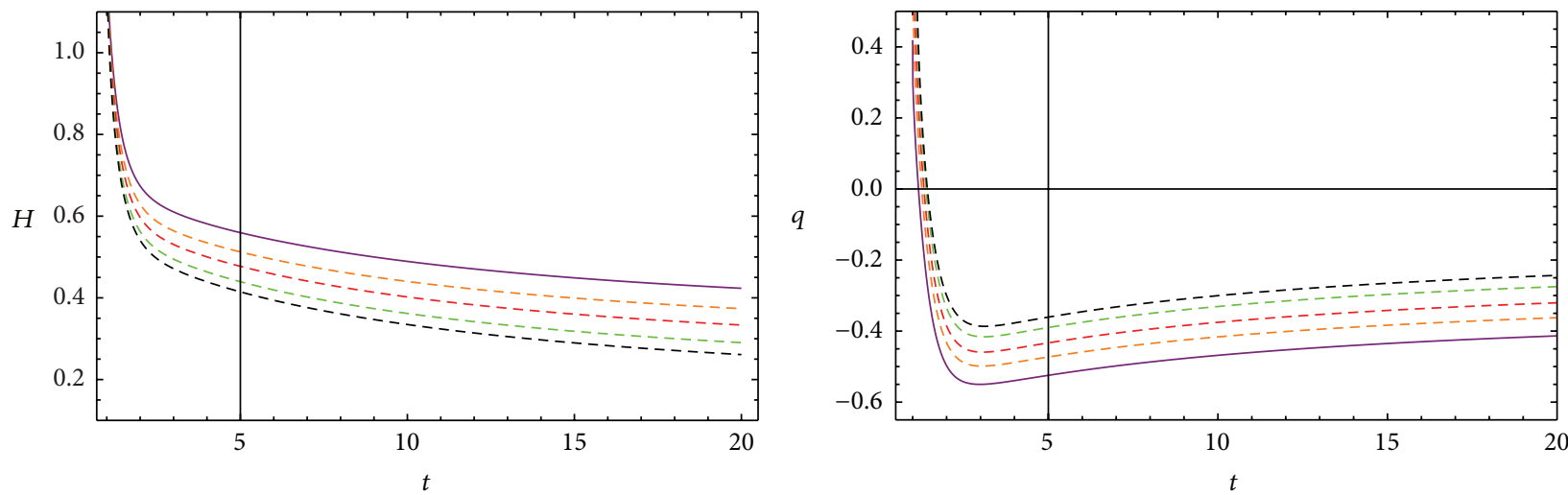

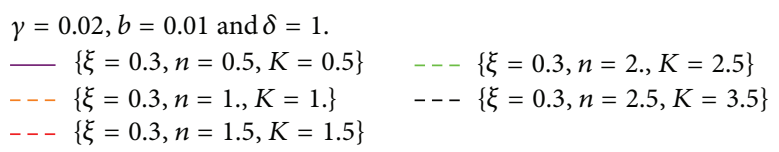

(a)

$$
\begin{aligned}
& \gamma=0.02, b=0.01 \text { and } \delta=1 \text {. } \\
& -\{\xi=0.3, n=0.5, K=0.5\} \quad--\{\xi=0.3, n=2 ., K=2.5\} \\
& --\{\xi=0.3, n=1 ., K=1 .\} \quad--\{\xi=0.3, n=2.5, K=3.5\} \\
& --\{\xi=0.3, n=1.5, K=1.5\}
\end{aligned}
$$

(b)

FiguRE 10: Behavior of Hubble parameter $H$ and deceleration parameter $q$ against $t$ for varying $\Lambda$. Model 5.

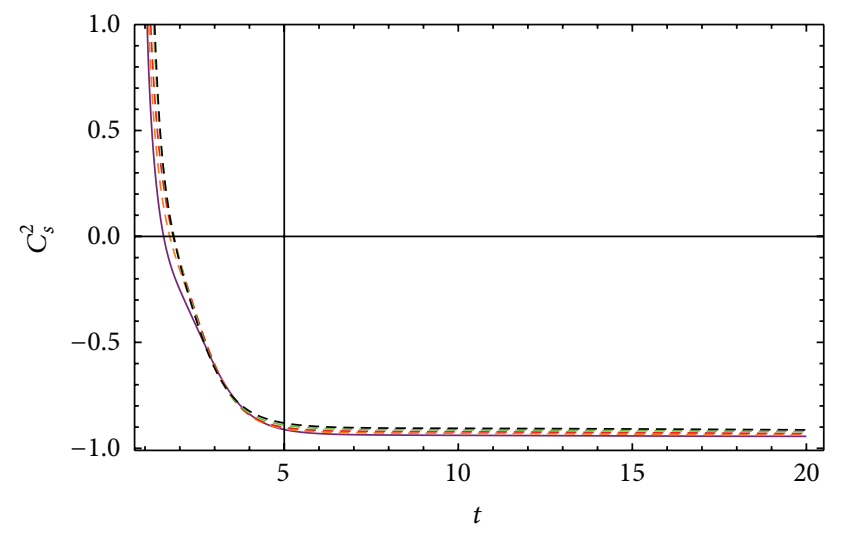

$\gamma=0.02, b=0.01$ and $\delta=1$.

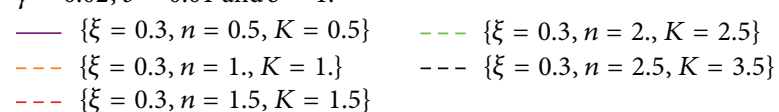

FIGURE 11: Squared sound speed against $t$ for varying $\Lambda$. Model 5 .

interacts with the quintessence dark energy, the differential equations describing the dynamics of the energy densities of both components are given:

$$
\begin{aligned}
\dot{\rho}_{W} & +3 H \rho_{W}\left(1+\frac{A}{B-\rho_{W}}-B \rho_{W}-\frac{\gamma}{3 H} \frac{\dot{\phi}}{\phi}\right) \\
& =3 H \rho_{\mathrm{Q}}\left(b-\frac{\gamma}{3 H} \frac{\dot{\phi}}{\phi}\right)+9 \xi H, \\
\dot{\rho}_{\mathrm{Q}} & +3 H \rho_{\mathrm{Q}}\left(1+b+\omega_{\mathrm{Q}}-\frac{\gamma}{3 H} \frac{\dot{\phi}}{\phi}\right)=-\gamma \rho_{i} \frac{\dot{\phi}}{\phi},
\end{aligned}
$$

where $\omega_{Q}$ is the EoS parameter of the dark energy. We obtain the behavior of important cosmological parameters as illustrated in Figure 12. Moreover, Figure 13 shows that this model is also instable at the late time.

Therefore we can choose the first model (model 4) as an appropriate model for the late time cosmology. Apart from the instabilities discussed above for the second and third models we can construct models by using more observational data discussed in the next section.

\section{Observational Constraints on Interacting Models with Varying $\Lambda$}

The SNIa test is based on the distance modulus $\mu$ which is related to the luminosity distance $D_{L}$ by

$$
\mu=m-M=5 \log _{10} D_{L},
$$

where $D_{L}$ is defined as

$$
D_{L}=(1+z) \frac{c}{H_{0}} \int_{0}^{z} \frac{d z^{\prime}}{\sqrt{H\left(z^{\prime}\right)}} \text {. }
$$

The quantities $m$ and $M$ denote the apparent and the absolute magnitudes, respectively. Baryonic acoustic oscillations have their origin in oscillations in the photon-baryon plasma at the moment of the decoupling at about $z=1.090$. They can be characterized by the distance scale

$$
A=\frac{\sqrt{\Omega_{m 0}}}{H\left(z_{b}\right)^{1 / 3}}\left[\frac{1}{z_{b}} \int_{0}^{z_{b}} \frac{d z}{H(z)}\right]^{2 / 3}
$$

The WiggleZ data [58] indicates the following information about $A$ and $z_{b}: A=0.474 \pm 0.034,0.442 \pm 0.020$, and $0.424 \pm 0.021$ for the redshifts $z_{b}=0.44,0.60$, and 0.73, 


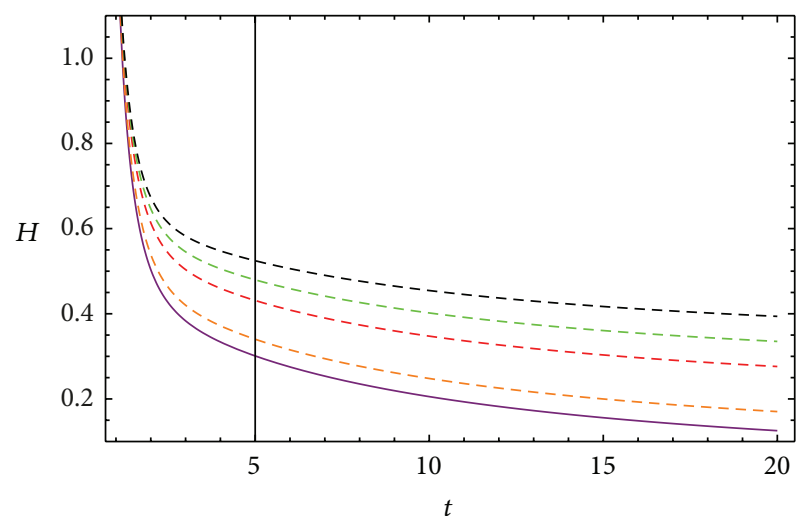

$$
\begin{gathered}
\gamma=0.02, b=0.01 \text { and } \delta=0.5 \\
-\{\xi=0 ., A=1 ., B=1.2\} \\
---\{\xi=0.1, A=1 ., B=1.2\} \\
---\{\xi=0.3, A=1 ., B=1.2\}
\end{gathered}
$$

$$
\begin{aligned}
& --\{\xi=0.4, A=1 ., B=1.2\} \\
& ---\{\xi=0.5, A=1 ., B=1.2\}
\end{aligned}
$$

(a)

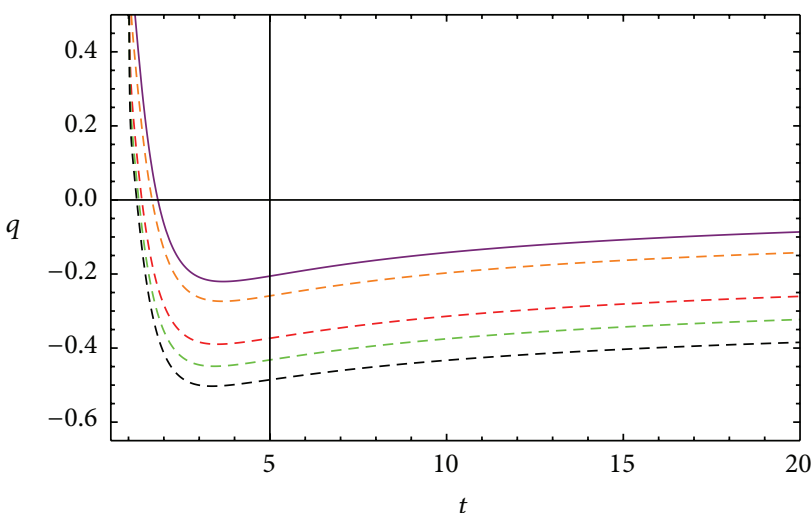

$\begin{aligned} & \gamma=0.02, b=0.01 \text { and } \delta=0.5 \\ &-\{\xi=0 ., A=1 ., B=1.2\} \\ &---\{\xi=0.1, A=1 ., B=1.2\} \\ &---\{\xi=0.3, A=1 ., B=1.2\}\end{aligned}$

$--\{\xi=0.4, A=1 ., B=1.2\}$

$--\{\xi=0.5, A=1 ., B=1.2\}$

(b)

FIGURE 12: Behavior of Hubble parameter $H$ and deceleration parameter $q$ against $t$ for varying $\Lambda$. Model 6 .

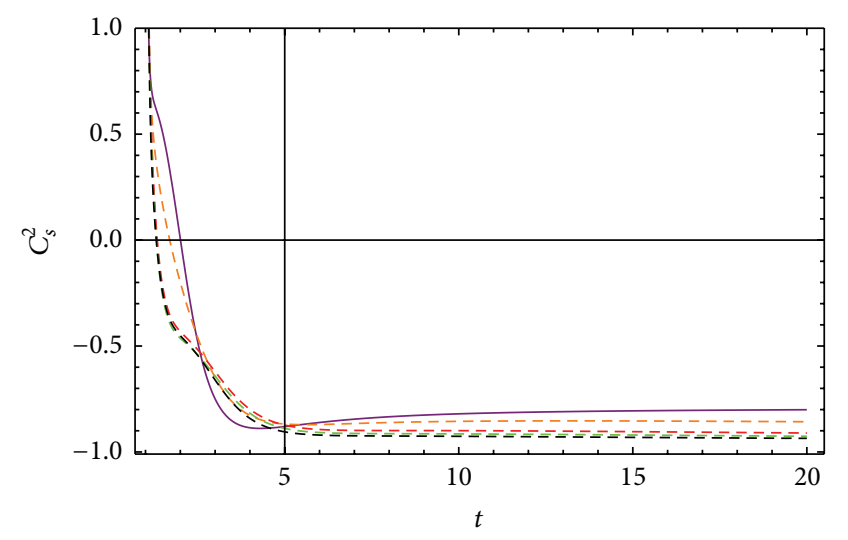

$\gamma=0.02, b=0.01$ and $\delta=0.5$

$-\{\xi=0 ., A=1 ., B=1.2\}$

$---\{\xi=0.1, A=1 ., B=1.2\}$

- - $\{\xi=0.3, A=1 ., B=1.2\}$

FIGURE 13: Squared sound speed against $t$ for varying $\Lambda$. Model 6 .

respectively. The key quantity of a statistical analysis is the $\chi^{2}$ parameter:

$$
\chi^{2}\left(x^{j}\right)=\sum_{i}^{n} \frac{\left(f\left(x^{j}\right)_{i}^{t}-f\left(x^{j}\right)_{i}^{0}\right)^{2}}{\sigma_{i}},
$$

where $f\left(x^{j}\right)_{i}^{t}$ is the theoretical evaluation of a given observable, depending on $x^{j}$ free parameters, $f\left(x^{j}\right)_{i}^{0}$ is the corresponding observational value, and $n$ is the total number of observational data for the given test. There are many different SNIa data sets, obtained with different techniques. In some cases, these different samples may give very different results. The second point is the existence of two different calibration methods. One of them uses cosmological relations
TABLE 1

\begin{tabular}{ccccccc}
\hline$M$ & $\delta$ & $\gamma$ & $b$ & $\xi$ & $H_{0}$ & $\Omega_{m 0}$ \\
\hline 4 & $1.3_{-0.2}^{+0.2}$ & $0.02_{-0.01}^{+0.03}$ & $0.01_{-0.01}^{+0.02}$ & $0.5_{-0.45}^{+0.25}$ & $1.1_{-0.2}^{+0.1}$ & $0.3_{-0.15}^{+0.15}$ \\
5 & $1.4_{-0.3}^{+0.2}$ & $0.02_{-0.01}^{+0.02}$ & $0.01_{-0.01}^{+0.02}$ & $0.3_{-0.15}^{+0.35}$ & $0.8_{-0.3}^{+0.2}$ & $0.25_{-0.1}^{+0.3}$ \\
6 & $0.75_{-0.15}^{+0.35}$ & $0.02_{-0.02}^{+0.03}$ & $0.03_{-0.02}^{+0.01}$ & $0.35_{-0.1}^{+0.15}$ & $1.4_{-0.1}^{+0.05}$ & $0.23_{-0.03}^{+0.03}$ \\
\hline
\end{tabular}

TABLE 2

\begin{tabular}{cccccc}
\hline$M$ & $A$ & $B$ & $\alpha$ & $K$ & $n$ \\
\hline 4 & $1.7_{-0.3}^{+0.2}$ & $0.3_{-0.15}^{+0.05}$ & $0.5_{-0.4}^{+0.2}$ & - & - \\
5 & - & - & - & $1.5_{-0.5}^{+0.3}$ & $1.2_{-0.3}^{+0.5}$ \\
6 & $1.2_{-0.1}^{+0.3}$ & $1.1_{-0.2}^{+0.4}$ & - & - & - \\
\hline
\end{tabular}

and takes into account SNIa with high $z$, and the other one, using astrophysical methods, is suitable for small $z$ (MLCS2k2). Our observational analysis of the background dynamics uses the following three tests: the differential age of old objects based on the $H(z)$ dependence as well as the data from SNIa and from BAO. A fourth test could potentially be added: the position of the first peak of the anisotropy spectrum of the cosmic microwave background radiation (CMB). However, the CMB test implies integration of the background equations until $z \approx 1.000$ which requires the introduction of the radiative component. But the inclusion of such radiative component considerably changes the structure of the equations and no analytic expression for $H(z)$ is available. Hence, we will limit ourselves to the mentioned three tests for which a reliable estimation is possible.

In Tables 1 and 2 we fix parameters of three models by using mentioned observational data.

In Figure 14, we can see the behavior of $\mu$ in all models, which is approximately in agreement with observational data. 


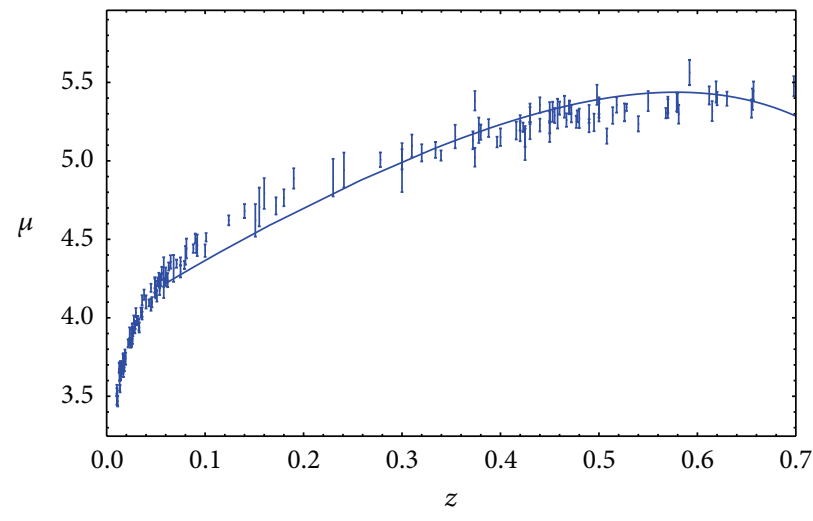

(a)

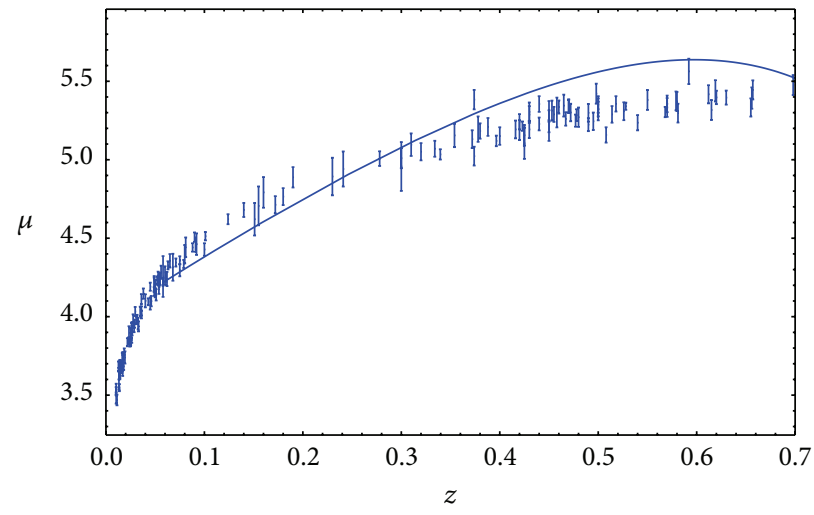

(b)

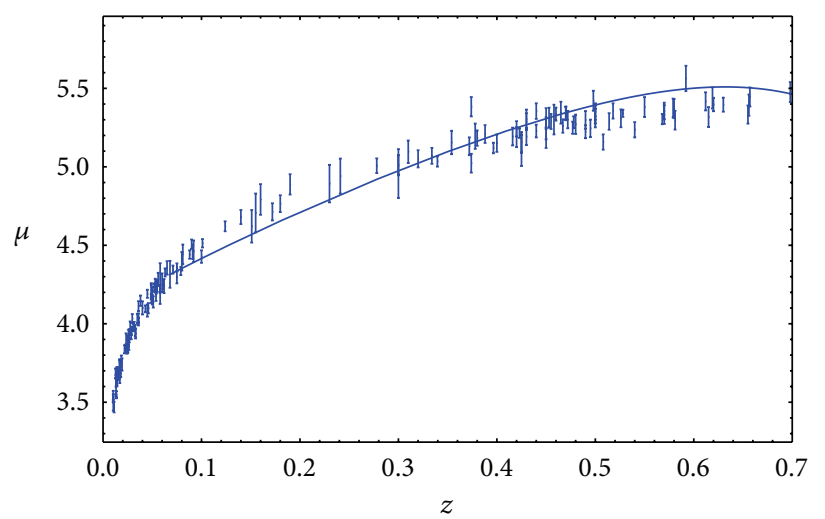

(c)

FIgURE 14: Observational data (SNeIa+BAO+CMB) for distance modulus versus our theoretical results for varying $\Lambda$ in models 4,5 , and 6 .

\section{Conclusion}

In this paper we considered three different cosmological models for the universe based on Lyra geometry. First of all we introduced our models and then obtained field equations solved numerically to find behavior of cosmological parameters. In order to find effect of varying $\Lambda$ we also studied the case of constant $\Lambda$ and found that presence of $\Lambda$ is necessary to obtain results being in agreement with observational data. We assumed viscous modified Chaplygin gas (models 1 and 4 ), viscous polytropic gas (models 2 and 5), or viscous Van der Waals gas (models 3 and 6) to be a component which at the early universe plays the role of dark matter with $\omega=0$, but at late times it tends to a cosmological constant. Moreover, we have a quintessence field, which will contribute to the dark energy sector including possibility of interaction between components. Easily we can check that $\Omega_{\mathrm{DE}}$ and $\Omega_{\mathrm{DM}}$ are of the same order. Also we considered case of varying $\Lambda$ and studied behavior of cosmological parameters numerically. We used observational data to fix parameters of the models and seen agreement with observational data by investigation of $H(z)$. By using stability analysis we concluded that the model is the best model considered in this paper to describe universe.

For the future work we can extend the present paper to include shear viscosity or varying bulk viscosity [53], and we can also consider cosmic Chaplygin gas versions [54] to obtain more general model.

\section{Conflict of Interests}

The authors declare that there is no conflict of interests regarding the publication of this paper.

\section{References}

[1] A. G. Riess, A. V. Filippenko, P. Challis et al., "Observational evidence from supernovae for an accelerating universe and a cosmological constant," The Astronomical Journal, vol. 116, no. 3, p. 1009, 1998.

[2] S. Perlmutter, G. Aldering1, G. Goldhaber et al., "Measurements of $\Omega$ and $\Lambda$ from 42 high-redshift supernovae," The Astrophysical Journal, vol. 517, p. 565, 1999.

[3] R. Amanullah, C. Lidman, D. Rubin et al., "Spectra and hubble space telescope light curves of six type Ia supernovae at $0.511<$ $z<1.12$ and the union2 compilation," The Astrophysical Journal, vol. 716, p. 712, 2010.

[4] A. C. Pope, T. Matsubara, A. S. Szalay et al., "Cosmological parameters from eigenmode analysis of sloan digital sky survey galaxy redshifts," The Astrophysical Journal, vol. 607, no. 2, pp. 655-660, 2004.

[5] D. N. Spergel, L. Verde, H. V. Peiris et al., "First year Wilkinson Microwave Anisotropy Probe (WMAP) observations: determination of cosmological parameters," The Astrophysical Journal Supplement, vol. 148, p. 175, 2003. 
[6] M. Jamil, K. Yesmakhanova, D. Momeni, and R. Myrzakulov, "Phase space analysis of interacting dark energy in $f(T)$ cosmology," Central European Journal of Physics, vol. 10, no. 5, pp. 10651071, 2012.

[7] W. Zimdahl, "Models of interacting dark energy," AIP Conference Proceedings, vol. 147, p. 51, 2012.

[8] M. Khurshudyan, B. Pourhassan, and E. Chubaryan, "A Universe with a generalized ghost dark energy and Van der Waals fluid interacting with a fluid," http://arxiv.org/abs/1302.1819.

[9] Y. L. Bolotin, A. Kostenko, O. A. Lemets, and D. A. Yerokhin, "Cosmological evolution with interaction between dark energy and dark matter," http://arxiv.org/abs/1310.0085.

[10] M. Khurshudyan, B. Pourhassan, and E. O. Kahya, "Interacting two-component fluid models with varying EoS parameter," International Journal of Geometric Methods in Modern Physics, vol. 11, no. 6, Article ID 1450061, 14 pages, 2014.

[11] P. P. Avelino and H. M. R. da Silva, "Effective dark energy equation of state in interacting dark energy models," Physics Letters B, vol. 714, no. 1, pp. 6-10, 2012.

[12] J. Sadeghi, M. Khurshudyan, A. Movsisyan, and H. Farahani, "Interacting ghost dark energy models in the higher dimensional cosmology," http://arxiv.org/abs/1401.6649.

[13] M. Khurshudyan, J. Sadeghi, A. Pasqua, S. Chattopadhyay, R. Myrzakulov, and H. Farahani, "Interacting Ricci dark energy models with an effective $\Lambda$-term in Lyra manifold," http://arxiv.org/abs/1402.5678.

[14] M. Khurshudyan, J. Sadeghi, M. Hakobyan, H. Farahani, and R. Myrzakulov, "Interaction between modified Chaplygin gas and ghost dark energy in the presence of extra dimensions," The European Physical Journal Plus, vol. 129, article 119, 2014.

[15] J. Sola and H. Stefancic, "Effective equation of state for dark energy: Mimicking quintessence and phantom energy through a variable $\Lambda$," Physics Letters B, vol. 624, no. 3-4, pp. 147-157, 2005.

[16] I. L. Shapiro and J. Solà, "On the possible running of the cosmological 'constant," Physics Letters B, vol. 682, no. 1, pp. 105-113, 2009.

[17] B. Ratra and P. J. E. Peebles, "Cosmological consequences of a rolling homogeneous scalar field," Physical Review D, vol. 37, no. 12, pp. 3406-3427, 1988.

[18] M. Khurshudyan, E. Chubaryan, and B. Pourhassan, "Interacting quintessence models of dark energy," International Journal of Theoretical Physics, vol. 53, no. 7, pp. 2370-2378, 2014.

[19] Z. K. Guo, N. Ohta, and Y. Z. Zhang, "Parametrizations of the dark energy density and scalar potentials," Modern Physics Letters A, vol. 22, no. 12, p. 883, 2007.

[20] S. Dutta, E. N. Saridakis, and R. J. Scherrer, "Dark energy from a quintessence (phantom) field rolling near a potential minimum (maximum)," Physical Review D, vol. 79, Article ID 103005, 2009.

[21] E. N. Saridakis and S. V. Sushkov, "Quintessence and phantom cosmology with nonminimal derivative coupling," Physical Review D, vol. 81, Article ID 083510, 2010.

[22] R. R. Caldwell, M. Kamionkowski, and N. N. Weinberg, "Phantom energy: dark energy with $w<-1$ causes a cosmic doomsday," Physical Review Letters, vol. 91, Article ID 071301, 2003.

[23] B. Feng, X. L. Wang, and X. M. Zhang, "Dark energy constraints from the cosmic age and supernova," Physics Letters B, vol. 607, no. 1-2, pp. 35-41, 2005.
[24] E. Elizalde, S. Nojiri, and S. D. Odintsov, "Late-time cosmology in a (phantom) scalar-tensor theory: dark energy and the cosmic speed-up," Physical Review D, vol. 70, Article ID 043539, 2004.

[25] Z. K. Guo, "Cosmological evolution of a quintom model of dark energy," Physics Letters B, vol. 608, pp. 177-182, 2005.

[26] M.-Z. Li, B. Feng, and X.-M. Zhang, "A single scalar field model of dark energy with equation of state crossing -1," Journal of Cosmology and Astroparticle Physics, vol. 2005, p. 002, 2005.

[27] B. Feng, M. Li, Y.-S. Piao, and X. Zhang, "Oscillating quintom and the recurrent universe," Physics Letters B, vol. 634, no. 2-3, pp. 101-105, 2006.

[28] S. Capozziello, S. Nojiri, and S. D. Odintsov, "Unified phantom cosmology: inflation, dark energy and dark matter under the same standard," Physics Letters, Section B, vol. 632, no. 5-6, pp. 597-604, 2006.

[29] W. Zhao and Y. Zhang, "Quintom models with an equation of state crossing -1," Physical Review D, vol. 73, Article ID 123509, 2006.

[30] Y. F. Cai, T. Qiu, Y. S. Piao, M. Li, and X. Zhang, "Bouncing universe with quintom matter," Journal of High Energy Physics, vol. 710, article 71, 7 pages, 2007.

[31] E. N. Saridakis and J. M. Weller, "A Quintom scenario with mixed kinetic terms," Physical Review D, vol. 81, no. 12, Article ID 123523, 11 pages, 2010.

[32] Y. F. Cai, T. Qiu, R. Brandenberger, Y. S. Piao, and X. Zhang, "On perturbations of quintom bounce," Joint Center for Artificial Photosynthesis, vol. 2008, no. 03, article 013, 2008.

[33] M. R. Setare and E. N. Saridakis, "Coupled oscillators as models of quintom dark energy," Physics Letters B, vol. 668, no. 3, pp. 177-181, 2008.

[34] M. R. Setare and E. N. Saridakis, "Quintom cosmology with general potentials," International Journal of Modern Physics D, vol. 18, no. 4, pp. 549-557, 2009.

[35] Y. F. Cai, E. N. Saridakis, M. R. Setare, and J. Xia, "Quintom cosmology: theoretical implications and observations," Physics Reports, vol. 493, no. 1, pp. 1-60, 2010.

[36] T. Qiu, "Theoretical aspects of Quintom models," Modern Physics Letters A, vol. 25, no. 11-12, pp. 909-921, 2010.

[37] Q. G. Huang and M. Li, “The holographic dark energy in a nonflat universe," Journal of Cosmology and Astroparticle Physics, vol. 8, 2004.

[38] M. Ito, "Holographic-dark-energy model with non-minimal coupling," Europhysics Letters, vol. 71, no. 5, pp. 712-716, 2005.

[39] X. Zhang and F. Q. Wu, "Constraints on holographic dark energy from type Ia supernova observations," Physical Review D, vol. 72, Article ID 043524, 2005.

[40] S. Nojiri and S. D. Odintsov, "Unifying phantom inflation with late-time acceleration: scalar phantom-non-phantom transition model and generalized holographic dark energy," General Relativity and Gravitation, vol. 38, pp. 1285-1304, 2006.

[41] E. Elizalde, S. Nojiri, S. D. Odintsov, and P. Wang, "Dark energy: vacuum fluctuations, the effective phantom phase, and holography," Physical Review D, vol. 71, Article ID 103504, 2005.

[42] H. Li, Z. K. Guo, and Y. Z. Zhang, "A tracker solution for a holographic dark energy model," International Journal of Modern Physics D, vol. 15, p. 869, 2006.

[43] E. N. Saridakis, "Restoring holographic dark energy in brane cosmology," Physics Letters B, vol. 660, no. 3, pp. 138-143, 2008. 
[44] E. N. Saridakis, "Holographic dark energy in braneworld models with moving branes and the $w=-1$ crossing," Journal of Cosmology and Astroparticle Physics, vol. 2008, no. 4, article 020, 2008.

[45] E. N. Saridakis, "Holographic dark energy in braneworld models with a Gauss-Bonnet term in the bulk. Interacting behavior and the $w=-1$ crossing," Physics Letters B, vol. 661, no. 5, pp. 335-341, 2008.

[46] R.-G. Cai, "A dark energy model characterized by the age of the universe," Physics Letters B, vol. 657, no. 4-5, pp. 228-231, 2007.

[47] H. Wei and R. G. Cai, "Interacting agegraphic dark energy," The European Physical Journal C, vol. 59, pp. 99-105, 2009.

[48] O. F. Piattella, J. C. Fabris, and W. Zimdahl, "Bulk viscous cosmology with causal transport theory," Journal of Cosmology and Astroparticle Physics, vol. 2011, no. 5, article 029, 2011.

[49] K. Karami, Z. Safari, and S. Asadzadeh, "Cosmological constraints on polytropic gas model," http://arxiv.org/abs/1209 .6374 .

[50] K. Karami, S. Ghaffari, and J. Fehri, "Interacting polytropic gas model of phantom dark energy in non-flat universe," European Physical Journal C, vol. 64, no. 1, pp. 85-88, 2009.

[51] K. Karami and S. Ghaffari, "The generalized second law of thermodynamics for the interacting polytropic dark energy in non-flat FRW universe enclosed by the apparent horizon," Physics Letters B, vol. 688, p. 125, 2010.

[52] G. M. Kremer, "Cosmological models described by a mixture of van der Waals fluid and dark energy," Physical Review D, vol. 68, no. 12, Article ID 123507, 2003.

[53] H. Saadat and B. Pourhassan, "Effect of varying bulk viscosity on generalized chaplygin gas," International Journal of Theoretical Physics, vol. 53, no. 4, pp. 1168-1173, 2014.

[54] B. Pourhassan, "Viscous modified cosmic chaplygin gas cosmology," International Journal of Modern Physics D, vol. 22, no. 9, Article ID 1350061, 2013.

[55] H. Saadat and B. Pourhassan, "FRW bulk viscous cosmology with modified Chaplygin gas in flat space," Astrophysics and Space Science, vol. 343, no. 2, pp. 783-786, 2013.

[56] H. Saadat and B. Pourhassan, "FRW bulk viscous cosmology with modified cosmic Chaplygin gas," Astrophysics and Space Science, vol. 344, no. 1, pp. 237-241, 2013.

[57] V. K. Shchigolev, "Cosmology with an effective $\Lambda$-term in Lyra Manifold," Chinese Physics Letters, vol. 30, Article ID 119801, 2013.

[58] M. Jamil, S. Ali, D. Momeni, and R. Myrzakulov, "Bianchi type I cosmology in generalized Saez-Ballester theory via Noether gauge symmetry," European Physical Journal C, vol. 72, no. 4, article 1998, pp. 1-6, 2012.

[59] M. Khurshudyan, "Toy models of Universe with an effective varying $\Lambda$-term," http://arxiv.org/abs/1403.0109. 

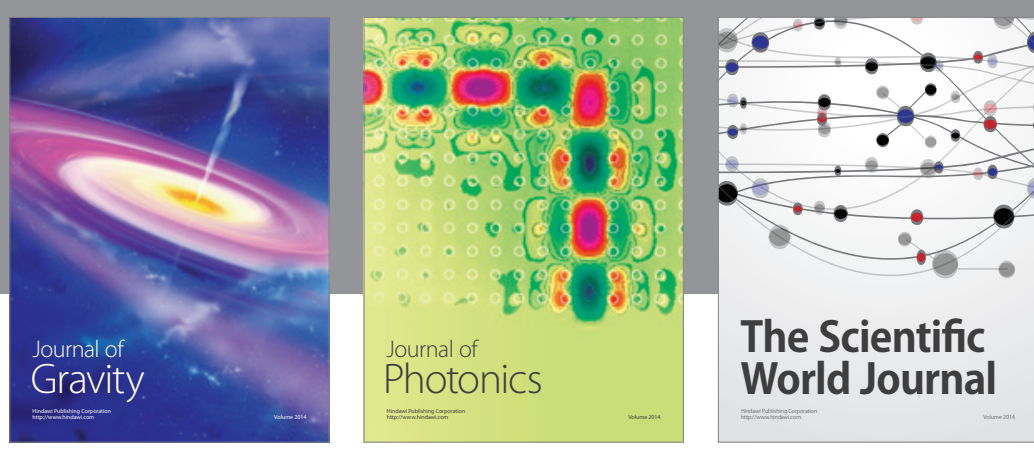

The Scientific World Journal
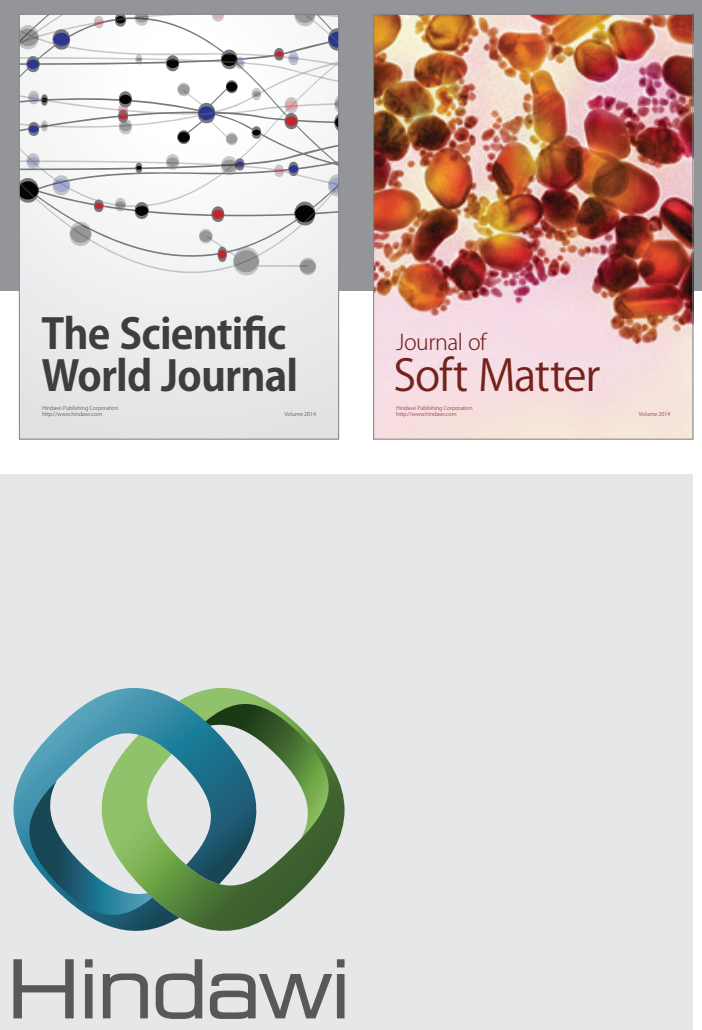

Submit your manuscripts at

http://www.hindawi.com

nternational Journal of

Statistical Mechanics
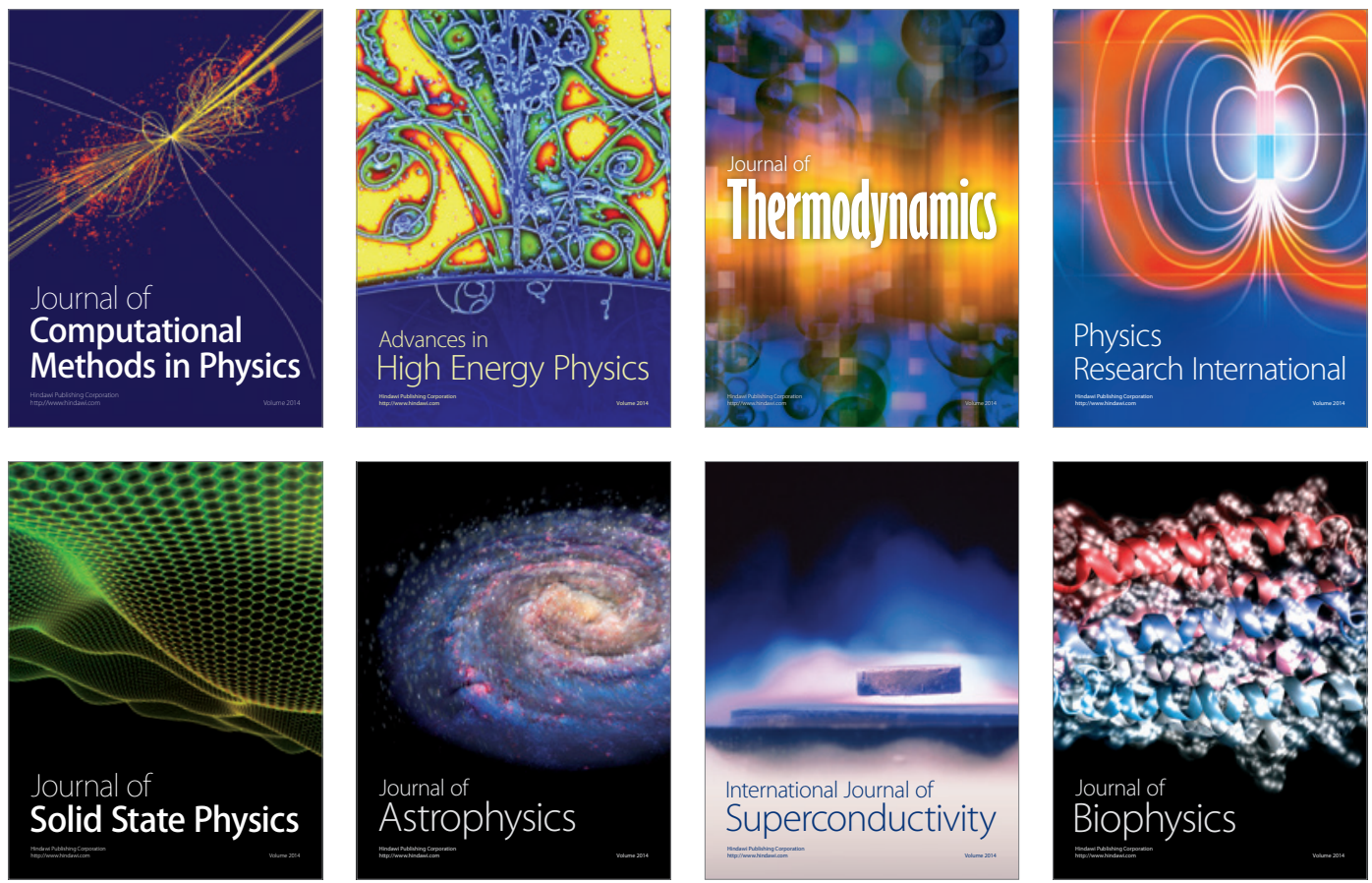
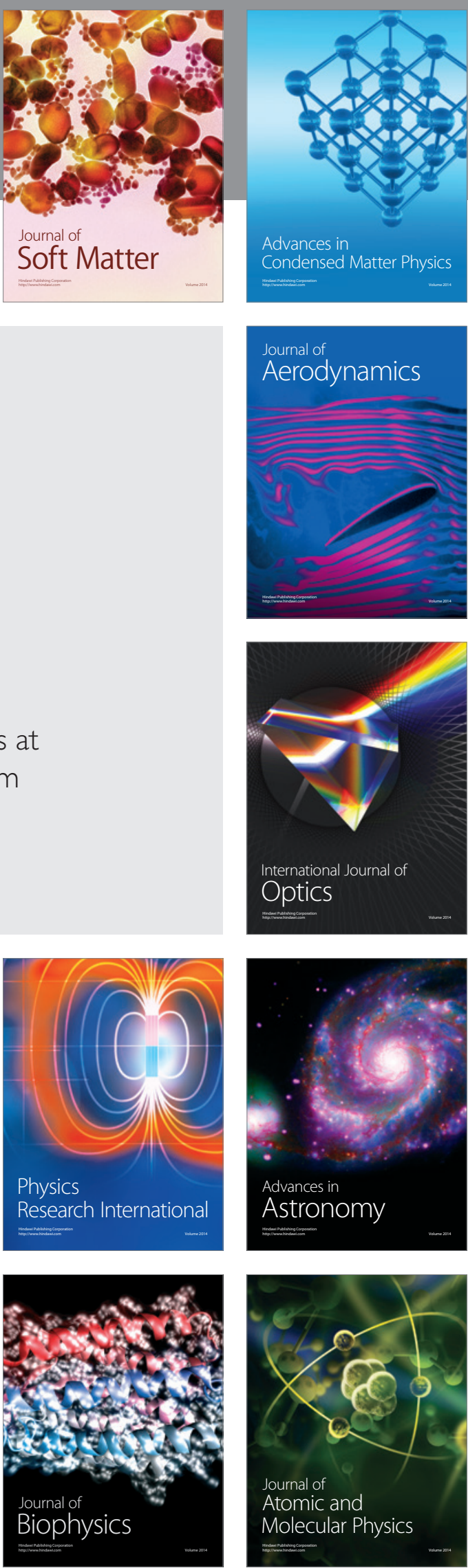